\title{
Contribuições da Reabilitação Neuropsicológica em Pacientes com Acidente Vascular Cerebral
}

\section{Contributions of Neuropsychological Rehabilitation in Patients with Cerebrovascular Accident}

\author{
Renan Gomes Lara
}

Universidade Candido Mendes, Programa de Pós-Graduação Stricto Sensu em Neuropsicologia. RJ, Brasil.

E-mail: reenamportales@gmail.com

\begin{abstract}
Resumo
Para compreender o termo reabilitação neuropsicológica se deve antes conceituar determinados termos como: identificar, recuperar e reorganizar, fatores esses que estão atrelados a determinada lesão cerebral que acometeu o indivíduo, como é o caso do Acidente Vascular Cerebral (AVC). O objetivo geral deste estudo foi identificar as contribuições da Neuropsicologia para o processo de reabilitação dos pacientes, que foram acometidos pelo Acidente Vascular Cerebral, bem como descrever as causas e consequências da lesão cerebral que comprometem os aspectos biopsicossociais, correlacionando com a prática clínica do neuropsicólogo. O delineamento da pesquisa foi do tipo qualitativo, descritivo, exploratório, buscando aprofundar a temática por meio de produções acadêmicas já elaboradas. Analisando os resultados encontrados dos pacientes, muitos apresentam lapsos de memória, mesmo após os três anos da sua ocorrência, levando a sérios problemas como o bradipsiquismo que é comum em pacientes com transtorno depressivo, esse fator se estima que os $35 \%$ dos doentes no início do processo de reabilitação podem apresentar esse estado patológico. Porém, através dos programas de reabilitação têm alcançado resultados significativos, como é o caso da exploração de ambientes virtuais e realidades virtuais, que corroboram para a validade ecológica, ou seja, desenvolvem novas estruturas de aprendizagem para o cérebro, promovendo assim o desempenho satisfatório e a melhora da qualidade de vida do indivíduo, contribuindo também no processamento da memória de trabalho.
\end{abstract}

Palavras-chave: Lesão Cerebral. Memória. Aprendizagem. Neuropsicologia.

\begin{abstract}
To understand the term neuropsychological rehabilitation, one must first conceptualize certain terms such as: identifying, recovering and reorganizing, factors that are linked to a certain brain injury that affected the individual, as is the case of a stroke. The general objective of this study was to identify the Neuropsychology contributions to the rehabilitation process of patients who were affected by a Stroke, as well as to describe the causes and consequences of brain injury, which compromise the biopsychosocial aspects, correlating with the neuropsychologist's clinical practice. The research design was of a qualitative, descriptive, exploratory type, seeking to deepen the theme through academic productions already prepared. Analyzing the results found from the patients, many have memory lapses, even after the three years of its occurrence, leading to serious problems such as bradypsychism that is common in patients with depressive disorder, this factor is estimated that $35 \%$ of the patients in the beginning of the rehabilitation process may present this pathological state. However, through rehabilitation programs they have achieved significant results, as it is the case of exploring virtual environments and virtual realities that corroborate the ecological validity, that is, they develop new learning structures for the brain, thus promoting satisfactory performance and improvement of the individual's quality of life, also contributing to the working memory processing.
\end{abstract}

Keywords: Brain Injury. Memory. Learning. Neuropsychology.

\section{Introdução}

O estudo da ciência neuropsicológica teve o início logo após as Primeiras Grandes Guerras Mundiais, os estudiosos da época buscavam mapear os diferentes tipos de lesões, de perdas cognitivas, ou danos cerebrais, apresentados pelos soldados sobreviventes das guerras, analisando também o impacto destrutivo causado pelo evento ao comportamento humano (PONTES; HÜBNER, 2008).

Nesse sentido, os processos da reabilitação neuropsicológica devem abranger alguns conceitos, tais como: identificar os possíveis déficits cognitivos que a doença comprometeu tomando como emergência a recuperação da resposta ao novo desempenho. Por outro lado, a reorganização funcional da disfunção cerebral pode ser atribuída às novas mudanças neuroplásticas, geradas por meio de treino cognitivo e da identificação precoce da área lesionada, antes avaliada pelo profissional com o objetivo de traçar uma nova forma de manejo clínico, a fim de contribuir para a melhora das funções executivas (CUNHA et al., 2007).

Os pesquisadores atribuem os estudos da neurociência para possíveis soluções e tratamentos para doenças, problemas e lesões no cérebro, como: Alzheimer, Parkinson, Acidente Vascular Cerebral, dor crônica, autismo, depressão, tumores cerebrais e lesões da medula espinhal (WELFERINGER, 2017).

Este estudo é de grande relevância para o cenário atual 
da Neuropsicologia brasileira, fazendo notória a investigação detalhada dos múltiplos fatores que alteram os aspectos neurológicos após o Acidente Vascular Cerebral - AVC. O processo da reabilitação neuropsicológica é um dos fatores fundamentais durante o processo de reabilitar o indivíduo, a psicologia nesse contexto contribui também através das técnicas de reeducação, de treinamento, de psicoterapia. Tornase fundamental a divulgação dessa área de conhecimento, que tem muito a ser explorada e ampliada para uma melhora do quadro clínico do doente em nível biopsicossocial (CRUZ et al., 2018).

De modo abrangente, a pesquisa busca compreender o trabalho realizado pelo profissional da neuropsicologia, que através das técnicas de reabilitação obtiveram uma melhora significativa ao quadro clínico dos pacientes que foram acometidos pelo AVC, ou seja, busca ressaltar as principais consequências que a lesão cerebral causa ao ser humano, assim como visa esclarecer os múltiplos fatores que a doença acarreta para a vida do indivíduo.

A problemática dos fatores relacionados ao AVC causa grande limitação para as funções executivas do indivíduo, como das áreas de processamento da informação, desenvolvimento cognitivo, linguagem e da motricidade. Desse modo, a neuropsicologia como ciência busca ampliar por meio de um leque de possibilidade proporcionar e auxiliar o doente através das técnicas, métodos e instrumentos terapêuticos (CORRÊA, 2009).

O objetivo geral deste estudo foi identificar as contribuições da Neuropsicologia para o processo de reabilitação dos pacientes, que foram acometidos pelo Acidente Vascular Cerebral (AVC), bem como descrever as causas e consequências da lesão cerebral que comprometem os aspectos biopsicossociais, correlacionando com a prática clínica do neuropsicólogo.

\section{Desenvolvimento}

\subsection{Metodologia}

O delineamento da pesquisa foi do tipo qualitativo, descritivo, exploratório, buscando aprofundar a temática por meio de produções acadêmicas já elaboradas, por meio de livros, artigos, monografias de especialização, dissertação de mestrado atualizados sobre o tema abordado em bases de dados e sites especializados como: Google Acadêmico, Scientific Electronic Library Online (SciELO). Desse modo, trata-se de uma revisão de literatura descritiva, a fim de conceituar a neuropsicologia; descrever as características do acidente vascular cerebral; compreender a atuação do neuropsicólogo clínico junto ao doente; revisar técnicas e métodos que auxiliam a reabilitação neuropsicológica.

Assim, o objetivo se direciona a descrever quais as consequências que a lesão causa na vida do indivíduo e, os principais fatores negativos relacionados a essa lesão cerebral que comprometem a vida em níveis biopsicossociais. A revisão integrativa busca incorporar resultados encontrados por meio do delineamento da pesquisa, ou seja, a síntese bibliográfica expõe a luz do conhecimento aos objetivos traçados no planejamento do estudo. Os descritores selecionados foram acidente vascular cerebral, neuropsicologia, reabilitação neuropsicológica, psicologia, tal como atuação neuropsicológica, instrumentos neuropsicológicos, técnicas e métodos, programas de reabilitação neuropsicológicos.

\subsection{A História da Neuropsicologia}

A Neuropsicologia é uma área de estudo das neurociências que visam compreender os aspectos biopsicossociais do ser humano. As pesquisas tiveram início logo após as Primeiras Grandes Guerras Mundiais, nesse sentido, os estudiosos da época buscavam mapear os diferentes tipos de lesões, de perdas cognitivas, ou de danos cerebrais, apresentados pelos soldados sobreviventes da Guerra, analisando também o impacto negativo causado pelo evento ao comportamento humano (PONTES; HÜBNER, 2008).

A psicologia dentro desse campo de estudo vem contribuindo, significativamente, para as novas descobertas da neurociência, atribuindo assim uma investigação detalhada, rigorosa e crítica dos fenômenos que permeia a natureza humana. Sendo assim, a ciência neuropsicológica pesquisa alguns distúrbios neurológicos causados ao cérebro, como exemplo, o acidente vascular cerebral (AVC), é uma doença que compromete as funções cognitivas e altera os aspectos comportamentais. O desenvolvimento de novas concepções epistemológicas e ontológicas são fatores fundamentais para que a Neuropsicologia tenha total respaldo científico, isto é, para as novas descobertas investigativas dos processos mentais superiores do ser humano (PESSOA, 2012). Vale ressaltar que no presente contexto a diferença entre reabilitação cognitiva e a neuropsicológica.

A publicação de Pontes e Hübner (2008, p.8) afirma que:

A reabilitação cognitiva visa "capacitar pacientes e familiares a conviver, lidar, contornar, reduzir ou superar as deficiências cognitivas resultantes de lesão neurológica", mas foca-se principalmente na melhora das funções cognitivas por meio dos treinos cognitivos. Já a reabilitação neuropsicológica é mais ampla, pois, além de almejar tratar os déficits cognitivos, objetiva também tratar as alterações de comportamento e emocionais, melhorando a qualidade de vida do paciente.

No entanto, é muito importante que o profissional observe por meio da avaliação neuropsicológica, qual é o grau de comprometimento apresentado pelo indivíduo, dessa forma objetiva traçar quais os cuidados necessários e as possíveis estratégias de reabilitação, a fim de preservar as funções cerebrais não afetadas, e contribuir para o impedimento das futuras sequelas de origem permanente que o paciente possa apresentar (FUENTES et al., 2014).

O estudo do Sistema Nervoso Central (SNC) está relacionado às áreas da neurociência que objetiva a pesquisar o mapeamento do cérebro entre os aspectos morfológicos, 
fisiológicos, processos mentais, organizações cerebrais e os possíveis distúrbios. No presente contexto, a Neuropsicologia é o campo de estudo entre duas grandes ciências: a psicologia e a neurologia, buscando atrelar um parâmetro entre o funcionamento cerebral e o comportamento, ou seja, ampliando o desenvolvimento dos recursos terapêuticos para uma melhor reabilitação neuropsicológica do paciente, que foi acometido por alguma doença, atuando assim nas funções mentais superiores (PAVAN et al., 2015). Portanto, tornase um campo amplo de estudo, a fim de encontrar possíveis respostas para as soluções e tratamentos para doenças, problemas e lesões no cérebro.

\subsection{Avaliação Neuropsicológica}

A Psicologia como ciência tem múltiplas ramificações que contribuem para a pesquisa e estudo do comportamento humano, como é o caso da Neuropsicologia que demanda atributos do profissional psicólogo voltados à área clínica e da psicometria, ambas disciplinas da prática psicológica. Nesse caso, o objeto da investigação será o sistema nervoso central (SNC) e, das possíveis causas patológicas que em decorrência de algumas doenças influenciou de modo positivo ou negativo o comportamento humano, assim a investigação ocorre através da avaliação neuropsicológica que compõem os recursos através de métodos, de técnicas e de abordagens psicoterapêuticas (CUNHA et al., 2007).

Entendida por Pontes e Hübner (2008, p.7):

[...] a análise do comportamento contribui com seus inúmeros procedimentos para a promoção da aprendizagem e mudanças comportamentais, oferecendo ao neuropsicólogo ferramentas valiosas, sobretudo a análise de contingências.

A avaliação neuropsicológica é uma investigação detalhada da demanda que foi solicitada pelo paciente, pois durante as sessões trará os subsídios ao profissional, por exemplo, o caminho a ser percorrido por meio de novas formas de atuação e possíveis tratamentos a serem seguidos, buscando dar uma possível resolução ao problema. Nesse sentido, o paciente que foi acometido por alguma lesão ou dano cerebral precisa de um possível prognóstico clínico que deva acompanhar os princípios fundamentais que espera desse profissional, que deve ser levado em consideração os aspectos emocionais, intelectuais e os biológicos, procurando adaptar e ampliar as funções da cognição que foram comprometidas após a lesão cerebral (SILVA; AREOSA, 2012).

Cunha et al. (2007) ressaltam os tópicos primordiais durante o processo da avaliação do paciente acometido pelo Acidente Vascular Cerebral, está em: identificar os déficits cognitivos que a doença comprometeu tomando como emergência a recuperação da resposta ao novo desempenho. No segundo tópico será a reorganização funcional da disfunção cerebral que pode contribuir para as mudanças neuroplásticas geradas através do treino cognitivo, dando o melhor manejo ao doente, assim proporcionando a melhora e recuperação gradual das funções executivas.
Nesse contexto, a reabilitação neuropsicológica em indivíduos que foram acometidos pelo acidente vascular cerebral está interligada com a prática do neuropsicólogo fundamentada entre os princípios técnicos e éticos, o principal meio para a investigação precisa da função afetada são os instrumentos neuropsicológicos que proporcionam a medida detalhada da lesão cognitiva. Segundo Silva (2010, p.6), a reabilitação:

[...] pretende capacitar os indivíduos com défices para melhorarem funções físicas, intelectuais, psicológicas e/ ou sociais. Compreende todo um programa durante o qual o doente progride para, ou mantém, o máximo grau de independência que é capaz.

Desse modo, a ciência neuropsicológica não deve apenas se ater a doença já instalada, mas procurar ampliar as mudanças em nível biopsicossocial do doente, pois o conjunto das práticas da reabilitação devem estar atreladas à recuperação das disfunções cognitivas lesionadas, da adaptação à nova realidade imposta pela doença, portanto, o comportamento do paciente também muda e sofre alteração durante o processo da recuperação que também pode acarretar sérios problemas psicológicos (CAMPOS, 2016). O papel do neuropsicólogo durante a reabilitação será de intervir por meio dos instrumentos, de técnicas e de métodos, porém sem deixar de lado a compreensão da singularidade e do respeito à valorização do outro.

\subsection{Acidente Vascular Cerebral (AVC)}

O Acidente Vascular Cerebral popularmente conhecido como (AVC) representa uma das causas de morte mundial, sendo um fator preocupante que impacta na qualidade de vida dos doentes, familiares e cuidadores, contribuindo para o afastamento laboral, e a perda da autonomia e dos afazeres domésticos (SILVA, 2010). Os inúmeros danos à saúde e os déficits cognitivos causados pelo AVC exigem uma intervenção interdisciplinar e multiprofissional especializada em diferentes áreas do conhecimento, a fim de proporcionar a recuperação gradual às áreas afetadas pela lesão.

Rolim e Martins (2011, p.2106) ressaltam que:

O AVC é classificado em dois grandes grupos: AVC isquêmico (AVCi) e o AVC hemorrágico. O mais frequente, com cerca de $85 \%$ dos casos, é o AVCi, que se caracteriza pela interrupção do fluxo sanguíneo (obstrução arterial por trombos ou êmbolos) em uma determinada área do encéfalo. No Brasil, o AVCi representa, na população nacional, segundo diferentes estatísticas, entre $53 \%$ a $85 \%$ dos casos de AVC.

O Acidente Vascular Cerebral hemorrágico tem por característica a ruptura de um vaso sanguíneo que causa grande fluxo de sangue e inchaço no local do cérebro (BRASIL, 2013). De acordo com Pavan et al. (2015, p. 833):

Os AVCs, sejam isquêmicos ou hemorrágicos, tendem a acarretar sequelas transitórias ou permanentes, tais como de saúde neurológica geral - prejuízos da função motora (paresias e plegias), sensitiva (parestesias) e do estado da consciência (coma) -, além de alterações neuropsicológicas, que podem 
ser cognitivas, comunicativas e/ou emocionais. Déficits cognitivos pós-AVC são muito frequentes e podem ter grande impacto no indivíduo e em sua família. Aproximadamente $65 \%$ dos pacientes que sobrevivem a um AVC apresentam prejuízos cognitivos. A ocorrência de alterações cognitivas está fortemente relacionada à dificuldade de recuperação do paciente e de seu benefício com a reabilitação, assim como à ocorrência de um segundo episódio de AVC.

No momento em que o indivíduo é acometido pelo acidente vascular cerebral, o processo hospitalar pode ser longo e exaustivo para o paciente e os familiares, a etiologia da doença é multifatorial e, na maioria dos casos, pode provocar sequelas neurológicas permanentes, portanto, o quadro clínico pode sofrer alterações de cunho psicológico (TERRONI et al., 2009). Os Acidentes Vasculares Cerebrais apresentam algumas características fundamentais das alterações em áreas específicas como: as funções executivas (a linguagem, a percepção, a memória, a atenção), observando também em alguns casos as alterações neuropsiquiátricas como é o caso da depressão e a ansiedade (PAVAN et al., 2015). Após a lesão cerebral já instalada, o profissional deve fazer a leitura detalhada em contexto clínico e, das múltiplas questões biopsicossociais envolvidas que podem surgir durante o tratamento.

\subsection{Reabilitação Neuropsicológica}

Os fundamentos que pautam a prática neuropsicológica demandam o interesse da comunidade científica, a fim de esclarecer dúvidas e contribuir para a melhora significativa dos doentes, e das diversas doenças de cunho neurológico. De acordo com Silva (2010, p.5):

Há três maneiras possíveis de o indivíduo com lesão cerebral poder recuperar as capacidades funcionais perdidas: recuperação espontânea, restituição ou compensação da função perdida [...].

Atribuindo os conceitos da reabilitação cognitiva, tais como: processamento de informação, de memória, de desenvolvimento cognitivo, de linguagem, entre outras, a reabilitação neuropsicológica nesse aspecto colabora, de modo que o profissional tenha uma maior abrangência de técnicas e métodos para poder reabilitar o doente.

Segundo a teoria, o sujeito passa a participar de seu processo de reabilitação de forma ativa, ou seja, como processador e transformador da informação, propiciando a modificabilidade cognitiva (CORRÊA, 2009, p.49).

Neste sentido, quando o profissional possui o conhecimento prévio da área cerebral mais lesionada, busca traçar os possíveis mecanismos de compensação que através do conjunto e do recurso terapêutico adequado passa a auxiliar todo o processo de reabilitação do doente, isso é, durante o treinamento, o cérebro está sendo estimulado, constantemente, e passa a desenvolver as novas conexões neurais, fenômeno conhecido como neuroplasticidade.

O Neuropsicólogo se torna um dos principais profissionais que trabalham para a melhora significativa do doente, seja nos aspectos físicos, mentais e sociais. Dessa forma, é visível a sua atuação como profissional da saúde. Fuentes et al. (2014, p.416) ressaltam que:

[...] É praticamente impossível reabilitar por completo um paciente neurológico, pois a maioria dos casos apresentam sequelas. Sendo assim, o terapeuta deve buscar ajustá-lo ao seu ambiente, considerando sua atual condição, de maneira a reintegrá-lo à sociedade e torná-lo o mais independente possível.

Os Programas de Reabilitação Neuropsicológicos (PRN) funcionam como um instrumento que visa corrigir as funções cognitivas afetadas em decorrência de alguma doença como é o caso do Acidente Vascular Cerebral. Nesse sentido, os métodos da reabilitação devem abranger a capacidade de recuperação e de potencialização das funções cerebrais afetadas, que por meio dos recursos terapêuticos adaptativos promovem a compensação, a adaptação e a reaprendizagem. A Neuropsicologia se torna uma área colaborativa da neurociência, que tem por objetivo buscar a melhora satisfatória do doente em âmbito biopsicossocial, e que através dos treinos cognitivos colaboram para a recuperação gradual das atividades neuronais (MURATORI; MORATORI, 2012).

\subsection{Psicologia e Psicoterapia}

De acordo com a publicação de Cruz et al. (2018, p. 94) é correto afirmar que:

[...] a psicologia tornou-se disciplina independente da filosofia e da fisiologia a partir do momento em que Wilhelm Wundt instituiu o primeiro laboratório de psicologia em Leipzig, na Alemanha. A teoria de Wundt era fundamentada na análise das sensações e sentimentos através do uso da concentração, processos extremamente subjetivos. Wundt acreditava que sujeitos instruídos adequadamente poderiam distinguir, exatamente, os processos mentais que acompanhavam sentimentos, sensações e pensamentos.

A psicologia compõe o leque de opções quando o assunto é o estudo dos fenômenos humanos, pois é uma ciência que tem por objetivo pesquisar como o cérebro pode influenciar, positiva ou negativamente o comportamento, e que através das recentes descobertas colaboraram com o meio científico nas últimas décadas, tal como propôs um novo campo de investigativo para a área da neurociência. Esse fato busca contextualizar a descoberta das novas abordagens psicoterapêuticas dentro da psicologia, sendo assim tem função de incorporar um maior arcabouço teórico e sustentar o conhecimento da ciência psicológica (PESSOA, 2012).

A psicologia se torna uma das áreas de conhecimento indispensável durante $\mathrm{o}$ processo da reabilitação neuropsicológica, pois durante todo o tratamento o paciente pode demandar conteúdo interno dentro da sessão terapêutica, o psicólogo especialista em neuropsicologia deve acolher o doente, compreendo através da escuta qualificada sua angústia e aflição, de modo único e singular, ou seja, “[...] é necessária uma integração de campos como psicoterapia, psicologia cognitiva, neuroplasticidade, linguística, psiquiatria, geriatria 
e outros campos" (PONTES; HÜBNER, 2008, p.7).

Scheffer, Klein e Almeida (2013, p.47):

[...] Dentre suas funções, a reabilitação neuropsicológica abrange uma combinação de psicoterapia, participação da família através de grupos, instruções terapêuticas aos pacientes, sendo sempre realizada dentro de um contexto multidisciplinar [...].

É sabido que a psicoterapia promove o ajustamento psicológico, fortalecimento social, boa capacidade cognitiva, criando os mecanismos de enfretamento a nova realidade que a doença impôs, dando assim uma maior chance de adaptação e de melhora da qualidade de vida (TERRONI et al., 2009).

A Neuropsicologia propõe evidenciar ativamente as novas formas de manejo dentro do setting terapêutico, traçando com a psicoterapia as questões da saúde mental do indivíduo, de modo que o paciente consiga passo a passo a sua recuperação. Assim, contribuir para uma maior investigação da complexidade humana em todos os seus aspectos correlacionando os processos mentais superiores através da adaptabilidade ao novo comportamento adquirido (PESSOA, 2012).

\subsection{Discussão da pesquisa}

A análise dos resultados encontrados foi feita por meio da pesquisa bibliográfica de artigos já publicados entre o período de 2013 a 2018. Desse modo, o objetivo traçado foi a identificação da Reabilitação Neuropsicológica (RN), em pacientes que sofreram o Acidente Vascular Cerebral - AVC, portanto, busca-se discutir as ideias de autores referência na área pesquisada. Os demais dados são encontrados no quadro a seguir.

Quadro 1 - Representação e a elaboração de artigos pesquisados

\begin{tabular}{|c|c|c|c|c|}
\hline Autor & Artigo & Objetivo & Metodologia & Resultado \\
\hline $\begin{array}{l}\text { Ferro; } \\
\text { Lins e } \\
\text { Trindade } \\
\text { Filho } \\
(2013)\end{array}$ & $\begin{array}{c}\text { Comprometimento } \\
\text { cognitivo e funcional em } \\
\text { pacientes acometidos de } \\
\text { acidente vascular encefálico: } \\
\text { Importância da avaliação } \\
\text { cognitiva para intervenção } \\
\text { na terapia ocupacional. }\end{array}$ & $\begin{array}{c}\text { Avaliar a capacidade } \\
\text { cognitiva de pacientes } \\
\text { que foram acometidos } \\
\text { pelo Acidente Vascular } \\
\text { Encefálico (AVE), } \\
\text { demostrando a importância } \\
\text { da avaliação cognitiva e } \\
\text { intervenção do TO. }\end{array}$ & $\begin{array}{l}\text { Delineamento transversal } \\
\text { e comparativo, com a } \\
\text { amostra composta de } 44 \\
\text { indivíduos, de ambos os } \\
\text { sexos. Divididos em grupos: } \\
\text { adulto; idoso e controle. }\end{array}$ & $\begin{array}{l}\text { Entre os grupos adulto e } \\
\text { idoso ambos apresentaram } \\
\text { alterações cognitivas, } \\
\text { porém, o grupo adulto } \\
\text { apresentou maior } \\
\text { comprometimento na } \\
\text { relação das atividades } \\
\text { cotidianas realizadas. }\end{array}$ \\
\hline $\begin{array}{l}\text { Scheffer; } \\
\text { Klein e } \\
\text { Almeida } \\
(2013)\end{array}$ & $\begin{array}{c}\text { Reabilitação } \\
\text { neuropsicológica em } \\
\text { pacientes com lesão } \\
\text { vascular cerebral: uma } \\
\text { revisão sistemática da } \\
\text { literatura }\end{array}$ & $\begin{array}{l}\text { Analisar determinados } \\
\text { programas de reabilitação } \\
\text { neuropsicológica, } \\
\text { ferramentas utilizadas em } \\
\text { pacientes com AVC. }\end{array}$ & $\begin{array}{l}\text { Buscou realizar uma } \\
\text { pesquisa do tipo revisão de } \\
\text { literatura em bases de dados } \\
\text { como: Web of Science, } \\
\text { Pubmed e Bireme entre os } \\
\text { anos de } 2000 \text { a } 2011 .\end{array}$ & $\begin{array}{c}\text { Os resultados mostraram } \\
\text { escassez de trabalhos tanto } \\
\text { na literatura internacional } \\
\text { e nacional, sendo voltado a } \\
\text { pesquisa a reabilitação da } \\
\text { linguagem. }\end{array}$ \\
\hline $\begin{array}{l}\text { Dantas } \\
\text { et al. } \\
(2014)\end{array}$ & $\begin{array}{c}\text { Rastreio cognitivo em } \\
\text { pacientes com acidente } \\
\text { vascular cerebral: um estudo } \\
\text { transversal }\end{array}$ & $\begin{array}{c}\text { Apontar o objetivo de fazer } \\
\text { o rastreio cognitivo em } \\
\text { paciente acometidos pelo } \\
\text { acidente vascular cerebral } \\
\text { (AVC). }\end{array}$ & $\begin{array}{c}\text { Estudo transversal com } 109 \\
\text { pacientes ambulatoriais } \\
\text { com a média de } 59 \text { anos. } \\
\text { Sendo que foram avaliados } \\
\text { pelo Mini Exame do Estado } \\
\text { Mental (MEEM). }\end{array}$ & $\begin{array}{c}\text { Apresentou uma } \\
\text { variedade de grau de } \\
\text { comprometimento } \\
\text { neurológico, idade e } \\
\text { escolaridade contribuíram } \\
\text { significativamente para o } \\
\text { valor global do MEEM. }\end{array}$ \\
\hline $\begin{array}{l}\text { Nunes; } \\
\text { Queirós } \\
\text { (2017) }\end{array}$ & $\begin{array}{c}\text { Doente com acidente } \\
\text { vascular cerebral: } \\
\text { planejamento de alta, } \\
\text { funcionalidade e qualidade } \\
\text { de vida }\end{array}$ & $\begin{array}{l}\text { Mostrar as estratégias } \\
\text { adotadas no planejamento } \\
\text { da alta hospitalar, tal como, } \\
\text { aprofundar à transição } \\
\text { hospital-domicílio. }\end{array}$ & $\begin{array}{c}\text { Buscou a revisão integrativa } \\
\text { de literatura, pelos critérios } \\
\text { orientadores PICOD, com } \\
\text { pesquisas em bases de } \\
\text { dados. }\end{array}$ & $\begin{array}{l}\text { Os } 19 \text { artigos pesquisados } \\
\text { tiveram diversas } \\
\text { abordagens e contextos. } \\
\text { Considerações dos aspectos } \\
\text { psicoemocionais, ligados à } \\
\text { funcionalidade. }\end{array}$ \\
\hline $\begin{array}{l}\text { Santos; } \\
\text { Teixeira e } \\
\text { Coelho } \\
(2018)\end{array}$ & $\begin{array}{c}\text { Neuropsicologia e } \\
\text { Reabilitação Cognitiva em } \\
\text { pacientes acometidos de } \\
\text { acidente vascular cerebral } \\
\text { encefálico }\end{array}$ & $\begin{array}{l}\text { Compreender o papel do } \\
\text { psicólogo nas atividades } \\
\text { que estimulem aspectos } \\
\text { cognitivos, apontando } \\
\text { exercícios de reabilitação. }\end{array}$ & $\begin{array}{l}\text { Pesquisa de cunho } \\
\text { bibliográfico e sistemático } \\
\text { sobre temas relacionados } \\
\text { a neuropsicologia e } \\
\text { reabilitação cognitiva de } \\
\text { pacientes com AVC. }\end{array}$ & $\begin{array}{l}\text { A neuropsicologia } \\
\text { busca traçar intervenção } \\
\text { através de estratégias } \\
\text { terapêuticas reabilitativas. } \\
\text { Já a reabilitação cognitiva } \\
\text { estimula as capacidades } \\
\text { funcionais cérebro-lesado. }\end{array}$ \\
\hline
\end{tabular}

Fonte: adaptado de Cruz et al. (2018).

De acordo com a publicação de Dantas et al. (2014), os pacientes acometidos pelo Acidente Vascular Cerebral (AVC), apresentam comprometimento cognitivo pelo menos até os três primeiros meses logo após o acidente cerebral, e continuam apresentando os lapsos da memória, também três anos depois da sua ocorrência, levando a sérios problemas como o bradipsiquismo que é uma condição comum em pacientes com transtorno depressivo, estima-se que os $35 \%$ dos doentes no início do processo de reabilitação podem apresentar essa lentificação comprometendo a função sensório-motora, a percepção e a linguagem, limitando assim a relação ao âmbito biopsicossocial do doente. 
Ferro, Lins e Trindade Filho (2013) ressaltam que por meio dos testes do Miniexame do Estado Mental (MEEM), Teste do Relógio e Teste das Trilhas, podem auxiliar na investigação mais precisa das áreas afetadas pelo AVC. Integrando a avaliação neuropsicológica e mapeando a perda das funções cognitivas acometidos pelo acidente vascular, pois envolvem as subcategorias que representam as respostas verbais e não verbais dadas pelo paciente no momento da avaliação. Assim, os instrumentos buscam avaliar também as habilidades preservadas, como é o caso da memória visuoespacial, construtiva e, das funções executivas, assim é sabido que o acidente vascular cerebral pode também afetar de modo parcial ou total a atenção visual, habilidade grafomotora e flexibilidade mental.

Para Dantas et al. (2014), os instrumentos necessitam ser sensíveis e fidedignos, como é o caso do teste (MEEM) que é o instrumento muito utilizado para o rastreio cognitivo dos pacientes após o acometimento do AVC, ou seja, buscar avaliar a localização mais precisa da lesão, e das disfunções neurais que determinam o processamento cognitivo, da memória e das funções executivas, portanto, os profissionais devem levar em conta o nível de comprometimento e, dos demais fatores como: a idade, o sexo, os transtornos psicológicos, e suas comorbidades relacionadas.

O tratamento inicia dentro do hospital sob a supervisão médica, após a identificação da ocorrência do AVC, após o ocorrido será acionada a equipe multiprofissional que tem por objetivo a supervisão de maneira integral do paciente, de modo a seguir uma rigorosa rotina de treinos e avaliações do quadro clínico. O neuropsicólogo nesse sentido pode traçar o plano terapêutico em equipe, que deverá ser seguido a fim de diminuir o impacto negativo que a doença causou, como também vir a evitar possíveis (re)internações hospitalares. $\mathrm{O}$ cuidado deve ser prestado pelo profissional da saúde, através da escuta qualificada da queixa inicial, e o acolhimento integral do paciente que se encontra fragilizado no momento, de difícil adaptação à nova realidade que a doença impôs (NUNES; QUEIRÓS, 2017).

Dantas et al. (2014) avaliam outro preditor da doença, que contribui para o déficit cognitivo, sendo esse a idade avançada de alguns pacientes, que após o acidente vascular cerebral, pode vir a causar a demência na fase crônica. Portanto, o grau de comprometimento neurológico vai depender da área afetada pelo AVC, por exemplo, a casos em que a afasia afeta cerca de $3 \%$ dos indivíduos mais velhos do que em pacientes jovens, também outro fator importante é a síndrome da negligência unilateral (SNU), que pode aparecer logo após o acidente vascular cerebral, vindo acompanhado de grande limitação da direção, resposta ou orientação no lado oposto da lesão cerebral, que são os traços visíveis no paciente.

Ferro, Lins e Trindade Filho (2013) corroboram afirmando que as alterações cognitivas em grupos mais jovens está relacionada à habilidade visoespacial, construtiva e das funções executivas, isto é, são áreas que estão intrinsicamente atreladas ao desempenho das atividades diárias, dessa forma, durante o processo da reabilitação neuropsicológica devem ser observadas quais são as habilidades que podem ser compensadas durante as sessões do treino cognitivo. Assim se deve avaliar frequentemente o grau de resposta para determinada atividade mental apresentada pelo paciente, trabalhando o mecanismo da recompensa para objetivar o aumento e o grau de independência.

O estudo de Santos, Teixeira e Coelho (2018) salienta que durante o processo da reabilitação são fundamentais as estratégias compensatórias, para o bom ajustamento das funções cognitivas afetadas, o período do treino cognitivo é longo, prolongado e sistemático, assim as sessões podem vir a contribuir para a restauração funcional do cérebro, portanto, nesse aspecto as necessidades e as questões pessoais do paciente devem ser levadas em conta pelo profissional, uma vez que através dos recursos terapêuticos são criados novos mecanismos compensatórios. O hemisfério esquerdo, por exemplo, pode regular o comportamento e a função da fala, da escrita e do cálculo. Já o hemisfério direito é responsável pela organização, memória visual, atenção reflexa, entre outros. O lado direito também processa os conteúdos nãoverbais, como é o caso das experiências, das atividades diárias, das orientações das imagens espaço-temporais e das relações interpessoais. A recuperação e o ajustamento das funções estão atreladas com as estratégias iniciais que o neuropsicólogo utilizará para o mapeamento da localização neural afetada, contribuindo e recuperando a função cognitiva que foi prejudicada no momento do acidente.

A combinação das estratégias e dos treinamentos deve seguir o parâmetro da qualidade e da execução, Scheffer, Klein e Almeida (2013) expõem que o treino cognitivo também pode contribuir para os pacientes, a fim de trazer mudanças que em longo prazo criam as novas habilidades, recompensando as que foram perdidas no momento do acidente vascular cerebral. Os programas de reabilitação têm alcançado resultados significativos como é o caso da técnica e exploração de ambientes virtuais, das realidades virtuais que corroboram para a validade ecológica (novas estruturas de aprendizagem), promovendo o desempenho satisfatório tanto na saúde mental, como na qualidade de vida, contribuindo amplamente para o processamento da memória de trabalho.

Os Programas de Reabilitação Neuropsicológica (PRN) têm alcançado pontos positivos na recuperação e na melhora das estruturas de reorganização cerebral dos pacientes que se encontravam na fase crônica do AVC, e que através das baterias de testes neuropsicológicos possibilitaram mesurar e avaliar o processo de reabilitação dos doentes, assim se observa que é possível melhorar as áreas que foram afetadas pelo acidente, e com o passar do tratamento obtiveram novas respostas e habilidades, que foram aprendidas através do mecanismo de compensação, sendo assim, esse tratamento visa trazer uma melhora ao quadro clínico e a recuperação das funções executivas como a atenção, a percepção, a flexibilização e a 
linguagem (SCHEFFER; KLEIN; ALMEIDA, 2013).

Durante as sessões de reabilitação, a região cerebral é trabalhada constantemente, a fim de proporcionar o encontro de novos estímulos, quanto maior o nível de recursos terapêuticos e de treinamentos cognitivos, melhor serão os resultados apresentados pelo paciente, o treinamento de cada indivíduo é singular e deve conter todos os recursos através de imagens, de jogos, de tarefas de resolução de problemas, de audiovisuais. A melhora das funções executivas ocorre através do conjunto de técnicas e de métodos que são traçados pelos profissionais da saúde, que desenvolvem o trabalho, de maneira holística, envolvendo também áreas da psicologia clínica, por meio do treinamento cognitivo, psicoterapia individual, análise comportamental, psicoeducação, neurofeedback (SANTOS; TEIXEIRA; COELHO, 2018).

\section{Conclusão}

$\mathrm{O}$ ato de reabilitar o paciente não deve ser entendido apenas como um simples gesto de cuidado, mas está além disso, pois procura compreender todos os aspectos biopsicossociais do doente que sofreu o Acidente Vascular Cerebral (AVC), visando melhora significativa de perda cognitiva e lesão cerebral, sendo assim trabalhando o contexto emocional e a qualidade de vida desse ser humano que se encontra fragilizado. A Neuropsicologia é um campo de estudo das neurociências que busca encontrar resoluções para determinadas áreas do cérebro, que foram afetadas por alguma doença. Nesse sentido, o neuropsicólogo como profissional capacitado busca investigar detalhadamente a demanda que foi solicitada através da queixa inicial, com o objetivo de estruturar o cérebro apara um novo comportamento adquirido.

Entre as atribuições desse profissional se encontra a prática clínica, que é composta por recursos, métodos, técnicas e abordagens psicoterapêuticas, tomadas como questões fundamentais para a investigação detalhada e mapeamento cerebral. Sendo assim, o prognóstico durante o processo de reabilitação do doente deve ser atribuído em conjunto aos fatores que envolvem os aspectos físicos, intelectuais e sociais, recuperando gradativamente a autonomia no contexto social. A atuação profissional ocorre pela prática interdisciplinar e multidisciplinar contemplando os planos, as medidas e as propostas terapêuticas. É sabido que em alguns casos a patologia neurovascular vem associada com o transtorno depressivo e ansioso, portanto, não se deve ater apenas a doença já instalada, mas proporcionar o leque de recursos terapêuticos que possam vir a contribuir para a independência, autodeterminação e autonomia e a autoestima do paciente.

Conclui-se que a neuropsicologia está em diversas áreas de atuação, seja na área clínica ou hospitalar, trabalhando através dos planos e de recursos terapêuticos para melhor atender o doente, que em função de seu quadro clínico está vivenciando uma nova realidade imposta pela doença, assim o profissional atua proporcionando a melhora na qualidade de vida de familiares / cuidadores. Sugere-se que em novas pesquisas sejam exploradas novas temáticas abordadas, para a melhor análise de conteúdo através da visão dos familiares em relação à progressão do quadro clínico do paciente, como também dos profissionais que atuam em diversos campos de trabalho.

\section{Referências}

BRASIL. Ministério da Saúde. Secretaria de Atenção à Saúde. Departamento de Ações Programáticas Estratégicas. Diretrizes de atenção à reabilitação da pessoa com acidente vascular cerebral / Ministério da Saúde, Secretaria de Atenção à Saúde, Departamento de Ações Programáticas Estratégicas. Brasília: MS, 2013.

CAMPOS, L.A.M. Psicologia da personalidade. Rio de Janeiro: SESES, 2016.

CORRÊA, R.C.R. Uma proposta de reabilitação neuropsicológica através do programa de enriquecimento instrumental (PEI). Ciênc. Cognição, v.14, n.2, p.47-58, 2009.

CRUZ, D.T. Desafios contemporâneos e as questões biopsicossociais. São Paulo: All Print, 2018.

CUNHA, J.A. et al. Psicodiagnóstico- $V$. Porto Alegre: Artmed, 2007.

DANTAS, A.A.T.S.G. et al. Rastreio cognitivo em pacientes com acidente vascular cerebral: um estudo transversal. $J$. Bras. Psiquiatr., v.63, n.2, p.98-103, 2014. doi: https://doi. org/10.1590/0047-2085000000012.

FERRO, A.O; LINS, A.E.S; TRINDADE FILHO, E.M. Comprometimento cognitivo e funcional em pacientes acometidos de acidente vascular encefálico: importância da avaliação cognitiva para intervenção na Terapia Ocupacional. Cad. Ter. Ocup. UFSCar, v.21, n.3, p.521-527, 2013. doi: https:// doi.org/10.4322/cto.2013.054.

FUENTES, D. et al. Neuropsicologia: teoria e prática. Porto Alegre: Artmed, 2014.

LANGE, E. S. N. Contribuições à psicologia hospitalar: desafios e paradigmas. São Paulo: Vetor, 2008.

MURATORI, M.F.P.; MURATORI, T.M.P. Neurofeedback na reabilitação neuropsicológica pós-acidente vascular cerebral. Rev. Neurocienc., v.20, n.3, p.427-436, 2012. doi: https://doi. org/10.34024/rnc.2012.v20.8255.

NUNES, H.J.M; QUEIRÓS, P.J.P. Doente com acidente vascular cerebral: planeamento de alta, funcionalidade e qualidade de vida. Rev. Bras. Enferm., v.70, n.2, p.433-442, 2017. doi: https:// doi.org/10.1590/0034-7167-2016-0166.

PAVAN, L.S. et al. Avaliação neuropsicológica no acidente vascular cerebral: um estudo de caso. Distúrbios Comum, v.27, n.4, p.831-839, 2015.

PESSOA, R.C. A Neuropsicologia e o pensamento complexo: possíveis interfaces e novos diálogos. Rev. EDUCAmazônia, v.9, n.2, p.75-91, 2012.

PONTES, L.M.M; HÜBNER, M.M.C. A reabilitação neuropsicológica sob a ótica da psicologia comportamental. Rev. Psiq. Clín., v.35, n.1, p.6-12, 2008. doi: https://doi.org/10.1590/ S0101-60832008000100002.

ROLIM, C.L.R.C; MARTINS, M. Qualidade do cuidado ao acidente vascular cerebral isquêmico no SUS. Cad. Saúde Pública, v.27, n.11, p.2106-2116, 2011. doi: https://doi. org/10.1590/S0102-311X2011001100004.

SANTOS, M.F.R; TEIXEIRA, H.P; COELHO, L.P. 
Neuropsicologia e reabilitação cognitiva em pacientes acometidos de acidente vascular encefálico. Rev. Transformar, v.12, n.1, 2018.

SCHEFFER, M; KLEIN, L.; ALMEIDA, R.M.M. Reabilitação neuropsicológica em pacientes com lesão vascular cerebral: uma revisão sistemática da literatura. Av. Psicol. Latinoam., v.31, n.1, p.46-61, 2013.

SILVA, R.B.F; AREOSA, S.V.C. Avaliação psicológica: desafios e possibilidades para a psicologia contemporânea. Santa Cruz do Sul: EDUNISC, 2012.
SILVA, E.J.A. Reabilitação após o AVC. Porto: Universidade do Porto, 2010.

TERRONI, L.M.N. et al. Depressão pós-AVC: aspectos psicológicos, neuropsicológicos, eixo HHA, correlato neuroanatômico e tratamento. Rev. Psiq. Clín., v.36, n.3, p.100-108, 2009. doi: https://doi.org/10.1590/S010160832009000900006 .

WELFERINGER, M.R.R. Neuropedagogia. São Luís: UemaNet, 2017. 